\title{
Prolonged Sleep Restriction Affects Glucose Metabolism in Healthy Young Men
}

\author{
Wessel M. A. van Leeuwen, ${ }^{1,2}$ Christer Hublin, ${ }^{1}$ Mikael Sallinen, ${ }^{1}$ Mikko Härmä, ${ }^{1}$ \\ Ari Hirvonen, ${ }^{3}$ and Tarja Porkka-Heiskanen ${ }^{2}$
}

\author{
${ }^{1}$ Brain and Work Research Centre, Finnish Institute of Occupational Health, Topeliuksenkatu 41 a A, 00250 Helsinki, Finland \\ ${ }^{2}$ Department of Physiology, Institute of Biomedicine, University of Helsinki, PO Box 63, 00014 Helsinki, Finland \\ ${ }^{3}$ Centre of Expertise for Health and Work Ability, Finnish Institute of Occupational Health, Topeliuksenkatu 41 a A, \\ 00250 Helsinki, Finland
}

Correspondence should be addressed to Tarja Porkka-Heiskanen, porkka@cc.helsinki.fi

Received 31 July 2009; Revised 13 November 2009; Accepted 10 February 2010

Academic Editor: Deborah Suchecki

Copyright (C 2010 Wessel M. A. van Leeuwen et al. This is an open access article distributed under the Creative Commons Attribution License, which permits unrestricted use, distribution, and reproduction in any medium, provided the original work is properly cited.

\begin{abstract}
This study identifies the effects of sleep restriction and subsequent recovery sleep on glucose homeostasis, serum leptin levels, and feelings of subjective satiety. Twenty-three healthy young men were allocated to a control group (CON) or an experimental (EXP) group. After two nights of $8 \mathrm{~h}$ in bed (baseline, BL), EXP spent $4 \mathrm{~h}$ in bed for five days (sleep restriction, SR), followed by two nights of $8 \mathrm{~h}$ (recovery, REC). CON spent $8 \mathrm{~h}$ in bed throughout the study. Blood samples were taken after the BL, SR, and REC period. In EXP, insulin and insulin-to-glucose ratio increased after SR. IGF-1 levels increased after REC. Leptin levels were elevated after both SR and REC; subjective satiety remained unaffected. No changes were observed in CON. The observed increase of serum IGF-1 and insulin-to-glucose ratio indicates that sleep restriction may result in an increased risk to develop type 2 diabetes.
\end{abstract}

\section{Introduction}

Sleep is considered to be a restorative process with beneficial effects on many bodily systems, including the digestive system, the immune system, and the cardiovascular system. Yet in modern industrialized societies, voluntary restriction of sleep is getting increasingly common due to, for instance, increasing work demands and atypical working hours [1]. Moreover, partial loss of sleep is common among people who experience environmental or psychological stress, who have psychiatric or physical disorders or who participate in shift work [2]. The consequences of this chronic deficiency of sleep are numerous and include increasing amounts of accidents, both in traffic and at work, increased prevalence of certain diseases, and even increased mortality [2]. It is important to understand and elucidate the mechanisms through which sleep and health are related if we are to find ways to manage people with chronically restricted sleep.

Sufficient sleep is a key component in the regulation of energy metabolism. Several epidemiological studies have shown that habitual short sleep duration is correlated with an increased risk of developing obesity and diabetes $[3,4]$. Controlled laboratory studies, investigating the effects of prolonged sleep restriction on energy metabolism, are more scarce. Glucose tolerance has been shown to be impaired after six days of sleep restricted to four hours per night, compared to a condition in which participants were allowed twelve hours in bed per night for six days [5], which might contribute to the risk of developing type 2 diabetes. Furthermore, it has been shown that two nights of sleep restricted to four hours, compared to two nights of ten hours in bed, results in a reduction of the satiety hormone leptin, accompanied by increased hunger and increased serum concentrations of the orexigenic factor ghrelin [6], which might add to the risk of developing obesity.

Rodent studies on energy metabolism have mainly applied either a total sleep deprivation or a selective REM sleep deprivation design, which are both difficult to compare to a sleep restriction design. Everson and Crowley showed, in rats, that 15 days of sleep restriction suppress concentrations 
of IGF-1 and leptin [7], which was, interestingly, accompanied by weight loss. Bodosi and colleagues have shown, on the other hand, that $5 \mathrm{~h}$ sleep deprivation does not affect leptin concentrations but increases ghrelin concentrations [8].

In the present study, we simulated accumulating sleep restriction during five working days followed by two days of weekend recovery sleep and measured the changes in several metabolic parameters that occurred during this period, including glucose metabolism, serum leptin concentrations, and feelings of satiety.

\section{Materials and Methods}

2.1. Participants. Twenty-three healthy men, aged 19-29 (mean \pm SD $23.1 \pm 2.5$ ), participated in this study and were recruited by advertisements in local newspapers during a two-year time span. First, volunteers were screened during a telephone interview, followed by a thorough physical examination, blood tests (triglycerides, cholesterol, haemoglobin, creatinine, leukocytes, erythrocytes, haematocrit, TSH, ASAT, ALAT, MCV, MCH, and MCHC), and screening polysomnography. Participants' final eligibility was evaluated according to preset inclusion and exclusion criteria. Volunteers were excluded from participation for any of the following: an irregular sleep-wake schedule, regular naps, having either advanced or delayed sleep phase syndrome, insomnia or other sleep problems, loud snoring $>5$ nights/week, repeating apneas, excessive daytime sleepiness (Epworth Sleepiness Scale $>8$ ), restless legs at least once a month, a disorder that might become worse because of prolonged wakefulness (such as a severe mental disorder, epilepsy, and cardiac arrhythmia), excessive caffeine consumption ( $>5$ cups of coffee/day), excessive alcohol consumption ( $>15$ units/week; 1 unit $=11 \mathrm{~g}$ or $13.9 \mathrm{~mL}$ of alcohol), smoking, medication affecting the central nervous system during the last two weeks, any clinically relevant abnormality on blood tests, any other reason that health may be harmed because of if participating, apnea-hypopnea index $>20$, periodic limb movement index $>25$, epileptiform activity on the EEG, abnormal urinary drug screening, and having experienced a significant recent life event that could disturb sleep. In addition, volunteers were only included when fulfilling all of the following criteria: male aged 19-29, sleep latency in the evening $<20-30$ minutes, uninterrupted nocturnal sleep and if awakened no problem to fall asleep again, no chronic disease or symptom affecting sleep, no continuous medication, and willing and able to participate.

All participants reported habitual sleep duration of $7-$ 9 hours and a regular sleep-wake schedule. For at least one week prior to the experiment they completed sleep diaries and carried actigraphs in order to verify adherence to a regular sleep-wake schedule. One week prior to the start of the experiment, participants had an adaptation night in the sleep laboratory. The prestudy mean $( \pm$ SD) sleep duration was $6.88( \pm 0.58) \mathrm{h}$ in the control group and $7.05( \pm 0.80) \mathrm{h}$ in the experimental group. Participant's prestudy mean $( \pm S D)$ body mass index (BMI) was $23.24( \pm 2.39)$ in the control group and $23.25( \pm 2.70)$ in the experimental group.
2.2. Experimental Protocol. The protocol was approved by the ethical committee of the University Hospital of Helsinki District, and written confirmed consent was obtained from all participants. A 10-day experimental schedule (Figure 1) was executed at the Brain and Work Research Centre of the Finnish Institute of Occupational Health (FIOH). Altogether, participants spent ten consecutive nights in the sleep laboratory. Fifteen participants were randomly allocated to the experimental group (EXP), spent the first two nights $8 \mathrm{~h}$ in bed (baseline, BL; from 23:00 h to 07:00 h), followed by five nights of $4 \mathrm{~h}$ in bed (sleep restriction, SR; from 03:00 h to $07: 00 \mathrm{~h}$ ) and, finally, again three nights of $8 \mathrm{~h}$ in bed (recovery, REC). Eight participants were randomly allocated to the control group (CON) and spent $8 \mathrm{~h}$ in bed every night. Sleep during the daytime was not allowed, which was monitored by EEG recordings and a continuously present investigator. During waking, participants took part in a bigger experiment of our sleep laboratory involving the simulation of a workweek by a variety of cognitive and psychological tasks. Their main activities during the day included the repeated assessment of the psychomotor vigilance task (PVT), the Brain@Work Multitask, a saccade test, and the training and testing of memory and motor tasks. Moreover, saliva samples were provided ten times per day and blood pressure was assessed eight times a day.

Participants ate standardized meals at fixed times throughout the experiment: breakfast at $08: 00 \mathrm{~h}(600 \mathrm{kcal})$, lunch at $12: 30 \mathrm{~h}(800 \mathrm{kcal})$, dinner at $18: 30 \mathrm{~h}(700 \mathrm{kcal})$; snacks at $15: 30 \mathrm{~h}(300 \mathrm{kcal})$ and $21: 30 \mathrm{~h}(200 \mathrm{kcal})$. In addition, participants in EXP ate a piece of fruit (apple or orange) at $00: 30 \mathrm{~h}(50 \mathrm{kcal})$. Participants were not allowed to leave the building but could, during regular short breaks, leave the sleep and test room and visit a relax room with television and PC. Illuminance in the sleep and test room ranged from 150 to 400 lux, and in the relax room from 350 to 600 lux, the temperature in the rooms ranged from 19 to 23 degrees Celsius. Polygraphy and ECG were continuously measured.

2.3. Hormonal Measurements. Hormonal levels were assessed from blood samples that were taken before breakfast at 07:30 h after the second BL night, the fifth SR night, and the second REC night in EXP and corresponding nights in CON. Samples were analyzed by Medix Laboratories, Espoo, Finland for glucose, insulin, IGF1, and leptin. Before blood sampling, subjects were asked to rate their feeling of hungriness on a 1 to 5 scale $(1=$ very hungry, $5=$ very satiated). The saliva samples described above were analyzed for cortisol levels using a commercial kit assay (Salivary Cortisol, LIA, IBL, Hamburg, Germany).

2.4. Statistical Analysis. For both CON and EXP, mean values $\pm \mathrm{SD}$ were calculated for each experimental day, BL, SR, and REC. In addition, SR and REC values were expressed as percentages of each individual participant's $\mathrm{BL}$ value, that is, normalized. We have compared SR and REC values to BL values by applying paired $t$-tests for normally distributed differences and Wilcoxon signed ranks 


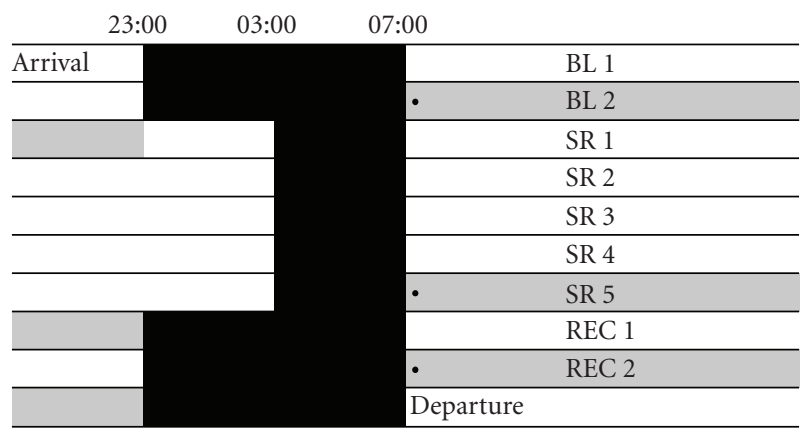

Figure 1: The experimental protocol. After two nights of $8 \mathrm{~h}$ sleep (baseline BL), sleep is restricted to $4 \mathrm{~h}$ per night for 5 subsequent days (sleep restriction, SR), followed by three nights of $8 \mathrm{~h}$ recovery sleep (REC). Profile days are shaded in grey. Bullet points indicate the taking of blood samples and rating of subjective satiety.

tests for differences that were not normally distributed. The normality of differences was checked using KolmogorovSmirnov goodness of fit test. A $P$-value $<.05$ was considered to be statistically significant. All statistical analyses were carried out using SPSS version 15 (SPSS Inc., Chicago, USA).

\section{Results}

3.1. Total Sleep Duration and Cortisol Profile. In CON, the mean total sleep duration $( \pm S D)$ remained unaffected throughout the experiment, whereas in EXP, the mean sleep duration, as expected, strongly reduced during the SR period (Table 1). In EXP, the peak in cortisol levels was delayed with $16.2 \pm 5.5 \mathrm{~min}$. after SR compared to BL $(P<.05$; Table 1$)$. After REC, the cortisol profile was similar to BL again. In $\mathrm{CON}$, the cortisol profile remained unaffected throughout the experiment (Table 1).

3.2. Glucose, Insulin, and IGF-1. Mean glucose, insulin, and IGF-1 levels throughout the experiment in both groups are described in Table 2. Glucose levels showed a tendency for a decrease after SR and its levels were significantly decreased after REC to $65.5 \% \pm 1.3 \%$ of BL levels $(P<.05$; Figure 2$)$. In $\mathrm{CON}$, glucose levels remained at $\mathrm{BL}$ level throughout the experiment (Figure 2).

Insulin levels were increased after SR to $159.9 \% \pm 25.6 \%$ of BL levels $(P<.05$; Figure 2$)$ and returned back to $\mathrm{BL}$ levels after recovery $(114.5 \% \pm 10.1 \%$ of $\mathrm{BL}$ levels $)$. In $\mathrm{CON}$, insulin levels remained at $\mathrm{BL}$ level throughout the experiment (Figure 2).

The insulin-to-glucose ratio was significantly increased after SR to $160.8 \% \pm 25.4 \%$ of BL levels $(P<.05$; Figure 2$)$, returning back to $\mathrm{BL}$ levels after subsequent REC $(118.2 \% \pm 9.9 \%$ of $\mathrm{BL}$ levels). In $\mathrm{CON}$, the insulin-toglucose ratio remained at $\mathrm{BL}$ level throughout the experiment (Figure 2).

IGF-1 levels showed a tendency for an increase after $\mathrm{SR}$ and its levels were significantly elevated after REC to $111.7 \% \pm 3.6 \%$ of BL levels $(P<.01)$. In CON, IGF-1 levels remained at BL level throughout the experiment (Figure 2).
3.3. Leptin and Subjective Satiety. Mean leptin levels and feelings of subjective satiety throughout the experiment in both groups are described in Table 2. Leptin levels were increased after SR to $163.3 \% \pm 42.4 \%$ of BL levels $(P<.01)$ and were still significantly elevated after REC $(123.1 \% \pm 7.0 \%$ of BL levels; $P<.01$; Figure 2$)$. In CON, leptin levels remained at $\mathrm{BL}$ level throughout the experiment (Figure 2).

Feelings of satiety remained unaffected throughout the experiment in both groups (Figure 2).

\section{Discussion}

Chronic sleep deprivation is becoming an increasingly common phenomenon in modern $24 \mathrm{~h}$ societies due to, for instance, voluntary sleep restriction and increasing work demands $[1,9]$. Restricted sleep does not only result in sleepiness and impaired cognitive performance, it also adversely affects general health [10]. Several widespread disorders have been shown to be epidemiologically associated with habitual short sleep duration, including cardiovascular diseases [11, 12], type 2 diabetes [13], and obesity [14, 15].

In the present study, serum glucose levels declined during the course of sleep restriction and subsequent recovery sleep, whereas serum insulin levels increased. Hence, the insulin-to-glucose ratio was significantly elevated after sleep restriction but returned to baseline values after subsequent recovery sleep. Elevating insulin levels that are not accompanied by elevations in glucose levels indicate a reduced sensitivity to insulin, which may ultimately increase the risk of developing noninsulin-dependent diabetes (i.e., type 2 diabetes). Taken together with a previous study showing that prolonged sleep restriction significantly lowers glucose tolerance [5], the experimental support for a causative connection between insufficient sleep and type 2 diabetes is gradually accumulating and supports the already present epidemiological evidence $[13,16,17]$.

Under normal physiological conditions, blood glucose concentrations are tightly regulated within narrow limits. A well-known condition in which blood glucose levels rise due to deficits in insulin signaling is diabetes, but no common conditions are known in which blood glucose levels decline. Blood glucose is the most important energy supply to the brain and, therefore, the observed decrease in glucose after recovery as compared to baseline is as puzzling as it is alarming, since the most important adverse effect of chronically decreased blood glucose levels is brain dysfunction and in extreme cases even damage [18]. Moreover, low levels of fasting blood glucose are associated with an increased mortality risk [19].

In addition to insulin, insulin-like growth factor-1 (IGF1 ) is another substance that lowers serum levels of glucose in both rats and humans $[20,21]$ and has even the capability of doing so in patients with severe insulin resistance [22]. The present study has indeed shown that, after recovery, IGF1 levels were increased while glucose levels were decreased. Interestingly, in addition to lowering serum glucose levels, IGF-1 has also been shown to decrease serum insulin levels in 


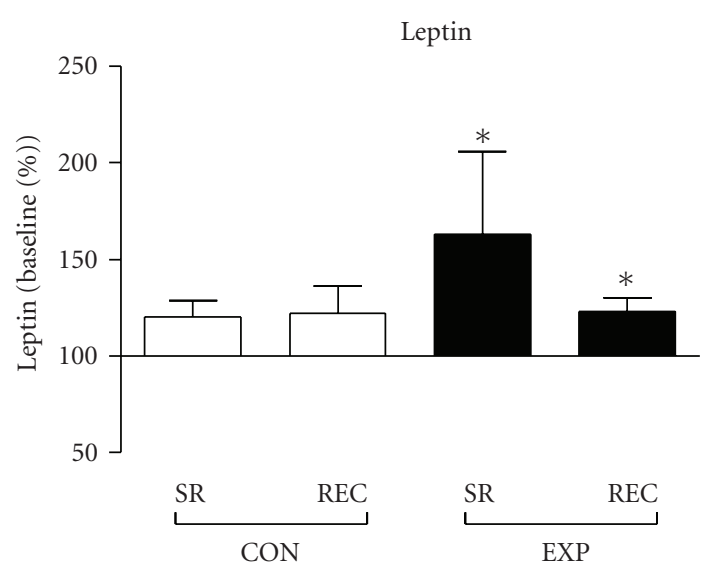

(a)

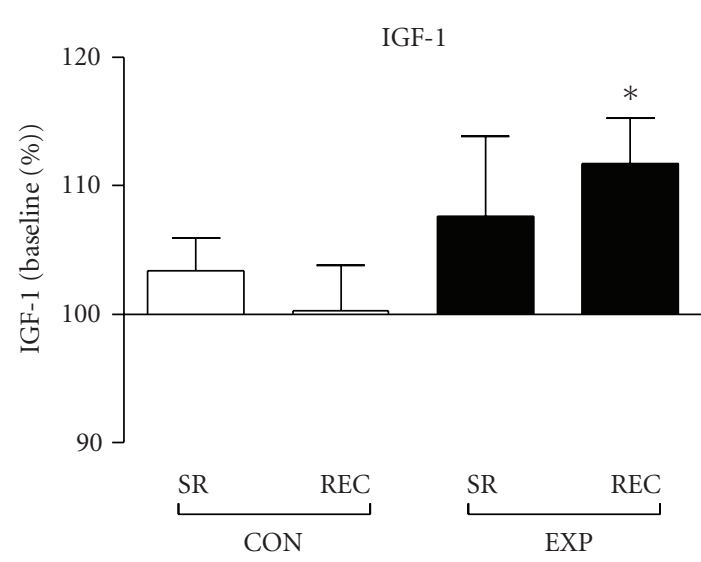

(c)

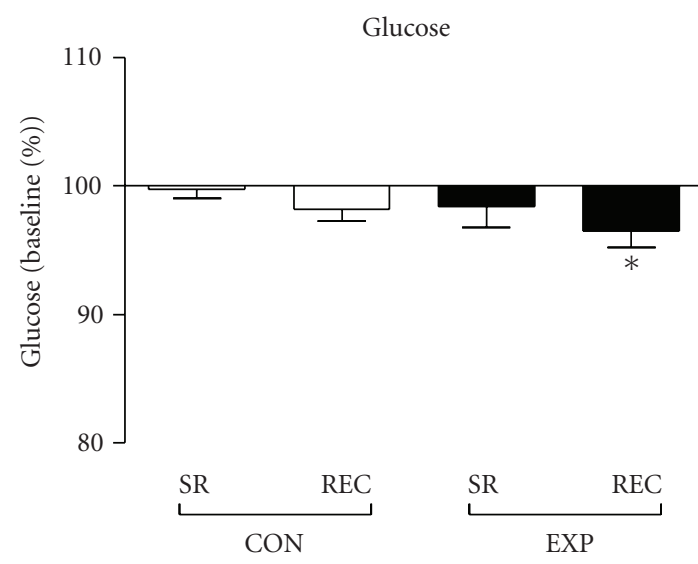

(e)

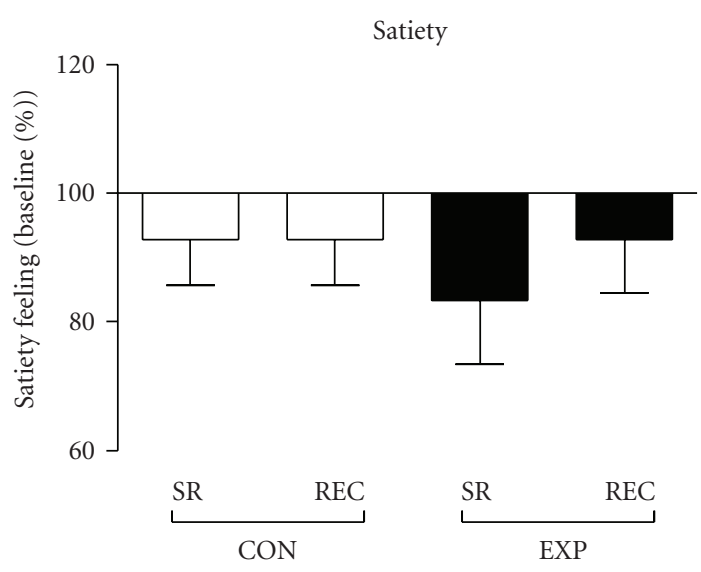

(b)

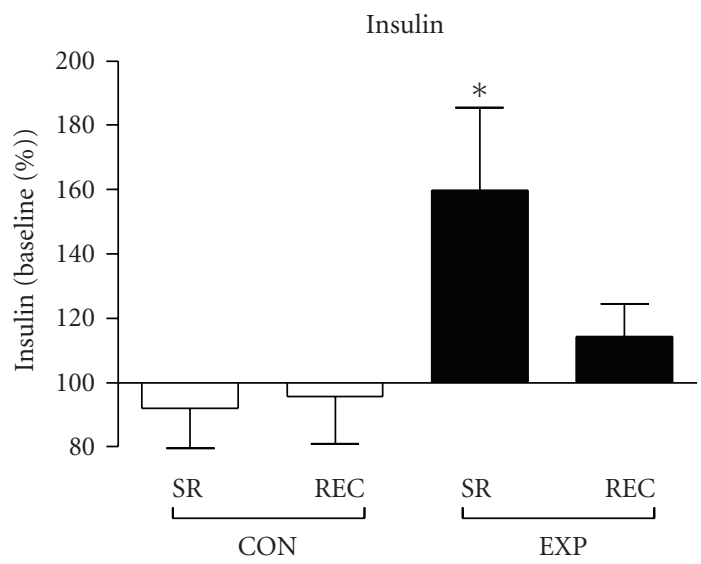

(d)

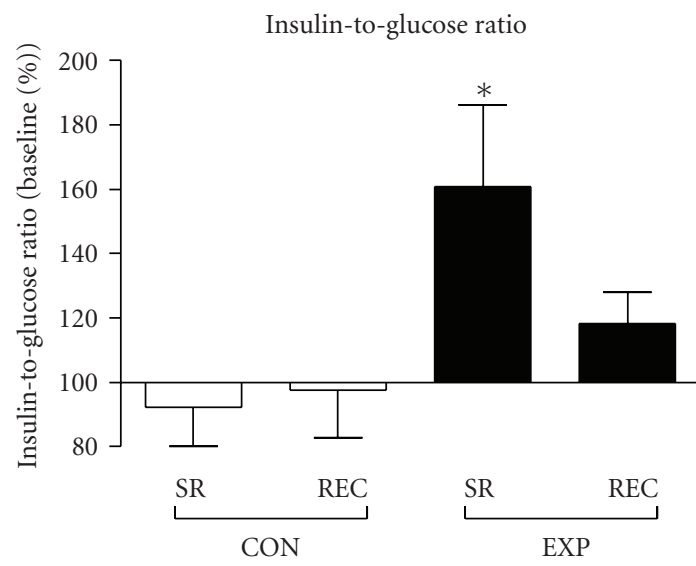

(f)

FIgURE 2: Changes in serum concentrations of leptin, IGF-1, insulin, and glucose, and changes in insulin-to-glucose ratio and subjective satiety after sleep restriction (SR) and recovery (REC) in the control group (CON) and experimental group (EXP). Data are expressed as percentages of participant's individual baseline values (mean \pm SEM) $\left({ }^{*} P<.05\right)$.

both rats and humans $[23,24]$. It has been hypothesized that, by lowering insulin levels, IGF-1 reduces insulin resistance and might thus be of therapeutical importance in physiological states that are associated with insulin resistance, such as type 2 diabetes. Hence, the observed elevations in IGF1 after recovery in the present study might be viewed as a compensatory reaction to the increased insulin levels after sleep restriction.

The rapidly expanding global incidence of obesity has a great impact on public health [25], for instance, by increasing the risk of developing cardiovascular diseases. Not only has this trend been paralleled by a trend of a gradual reduction 
TABLE 1: Sleep duration and cortisol.

\begin{tabular}{lcccc}
\hline Variable & Group & BL & Day & SR \\
& & Mean $( \pm$ SD $)$ & Rean $( \pm$ SD $)$ & $\begin{array}{c}\text { Rean }( \pm \text { SD }) \\
\text { Total sleep duration (min.) }\end{array}$ \\
& CON & $440( \pm 23)$ & $442( \pm 16)$ & $429( \pm 27)$ \\
& EXP & $439( \pm 20)$ & $232( \pm 5)$ & $458( \pm 13)$ \\
\hline \multirow{2}{*}{ Cortisol peak (clock time) } & CON & $07: 48( \pm 0: 15)$ & $07: 33( \pm 0: 19)$ & $07: 33( \pm 0: 10)$ \\
& EXP & $07: 39( \pm 0: 14)$ & $07: 55( \pm 0: 11)$ & $07: 36( \pm 0: 13)$ \\
\hline
\end{tabular}

TABLE 2: Descriptives of the data.

\begin{tabular}{|c|c|c|c|c|}
\hline \multirow{3}{*}{ Variable } & \multirow{3}{*}{ Group } & \multicolumn{3}{|c|}{ Day } \\
\hline & & $\mathrm{BL}$ & SR & REC \\
\hline & & Mean $( \pm$ SD $)$ & Mean $( \pm$ SD $)$ & Mean $( \pm$ SD $)$ \\
\hline \multirow{2}{*}{ Glucose (mmol/L) } & $\mathrm{CON}$ & $5.14( \pm 0.50)$ & $5.13( \pm 0.49)$ & $5.04( \pm 0.41)$ \\
\hline & EXP & $4.91( \pm 0.24)$ & $4.83( \pm 0.24)$ & $4.74( \pm 0.17)$ \\
\hline \multirow{2}{*}{ Insulin $(\mathrm{mU} / \mathrm{L})$} & $\mathrm{CON}$ & $8.27( \pm 2.62)$ & $7.33( \pm 2.84)$ & $7.50( \pm 2.82)$ \\
\hline & EXP & $6.02( \pm 2.90)$ & $8.59( \pm 6.99)$ & $6.25( \pm 2.36)$ \\
\hline \multirow{2}{*}{ Insulin-to-glucose ratio } & $\mathrm{CON}$ & $1.65( \pm 0.65)$ & $1.49( \pm 0.73)$ & $1.52( \pm 0.67)$ \\
\hline & EXP & $1.22( \pm 0.57)$ & $1.75( \pm 1.33)$ & $1.31( \pm 0.46)$ \\
\hline \multirow{2}{*}{ IGF-1 (nmol/L) } & $\mathrm{CON}$ & $33.81( \pm 11.15)$ & $34.84( \pm 10.99)$ & $34.03( \pm 11.63)$ \\
\hline & EXP & $26.35( \pm 6.29)$ & $27.64( \pm 5.39)$ & $28.93( \pm 5.23)$ \\
\hline \multirow{2}{*}{ Leptin $(\mu \mathrm{g} / \mathrm{L})$} & $\mathrm{CON}$ & $3.44( \pm 3.09)$ & $3.79( \pm 2.92)$ & $3.70( \pm 2.80)$ \\
\hline & EXP & $6.25( \pm 4.55)$ & $7.59( \pm 5.00)$ & $6.93( \pm 4.07)$ \\
\hline \multirow{2}{*}{ Satiety (1 to 5 scale) } & $\mathrm{CON}$ & $2.71( \pm 0.76)$ & $2.57( \pm 0.98)$ & $2.57( \pm 0.98)$ \\
\hline & EXP & $2.21( \pm 0.43)$ & $1.79( \pm 0.70)$ & $2.00( \pm 0.55)$ \\
\hline
\end{tabular}

in self-reported sleep duration, many epidemiological studies have linked those trends and observed a correlation between short sleep and obesity [26]. Recently, however, several groups have questioned the clinical relevance of this link $[27,28]$. Expanding the current literature with experimental investigations might attribute to resolving those heated debates, editorials, and news reports.

Leptin and ghrelin are peripheral hormones believed to contribute to the central regulation of food intake [29]. Ghrelin, predominantly released by the stomach, stimulates appetite whereas leptin, mainly produced by adipocytes, stimulates feelings of satiety. Therefore, chronic elevations of ghrelin levels and/or reductions of leptin levels may attribute to the development of obesity. Obesity is indeed associated with leptin resistance and obese subjects show highly elevated serum concentrations of leptin [30]. Hitherto, only two experimental studies have investigated the effects of prolonged sleep restriction on serum ghrelin and leptin levels and observed decreased leptin and increased ghrelin levels, accompanied by increased feelings of hunger and appetite after a period of $4 \mathrm{~h}$ sleep compared to a period of $10 \mathrm{~h}$ sleep [6] and $12 \mathrm{~h}$ sleep [31]. In addition, prolonged sleep restriction in rats has been shown to result in decreased leptin levels that were associated with a reduction in body weight despite an increase in food intake [7]. Our observations, interestingly, contradict those findings in not having found any changes in feelings of hunger and having found an increase rather than a decrease in serum leptin levels.
Several factors are known to regulate serum leptin concentrations. Taheri and colleagues have shown that sleep duration is correlated to leptin levels [32]. Hence, longsleepers have higher serum leptin concentrations than shortsleepers. We are, however, the first to show in a withinsubject design that experimental restriction of participant's habitual sleep duration does not have a similar effect and that it is even increasing serum leptin concentrations. The only previous experimental studies compared sleep restriction against sleep extension and have found that after sleep restriction, leptin levels are lower than after sleep extension but unaffected as compared to participant's habitual sleep duration $[6,31]$.

Serum leptin concentrations are known to exhibit a circadian rhythm, with minimum values during daytime and a nocturnal rise [33]. This rhythm is not entrained to the circadian clock, but to meal patterns [34]. However, it does not acutely change in response to single meals [35]: a substantial meal of $1000 \mathrm{kcal}$ did not alter leptin levels for the next three hours after administration [36]. In our study, there was only a modest (16 minute) delay in the endogenous circadian rhythm of salivary cortisol in the experimental group. However, meal timing was kept constant throughout the experiment, except for an additional apple or orange that participants in the experimental group received at 00:30 h during the days of restricted sleep. We find it unlikely that this small addition of about $50 \mathrm{kcal}$ for a period of five days to the habitual meal pattern would have increased leptin 
levels with $63 \%$. How abolishing oral meals completely by replacing them with intravenous glucose infusion affects serum leptin concentrations [6] is not known.

Physical activity is inversely related to fasting plasma leptin levels [37]. Physical exercise has indeed been shown to result in decreased concentrations of serum leptin, both acutely and over the entire $24 \mathrm{~h}$ time span $[38,39]$. In the present study, however, we did not aim to keep physical activity constant. Hence, participants in the experimental group were not restricted in their physical activity during the period of prolonged wakefulness. Therefore, physical activity was slightly elevated during this period when compared to the baseline and recovery periods. This could, in theory, have decreased leptin levels.

Leptin has for a long time been considered to be purely a satiety signal and previous sleep restriction studies have indeed found that lower leptin levels were associated with increased feelings of hunger [6]. In the present study, however, elevated leptin levels were not accompanied by any changes in hunger feelings. This may suggest that leptin plays additional physiological roles apart from regulating food intake, such as a proinflammatory role $[40,41]$.

An alternative explanation for the observed epidemiological correlation between short sleep and obesity might have little to do with the homeostatic control of sleeping and feeding behavior. As Saper and colleagues have pointed out, both the regulation of feeding and sleeping have a strong hedonic component $[42,43]$. That is, both can be very satisfying at times when their physiological need is not that strong. It may be that, under the unpleasant experience of restricted sleep, a search for pleasure begins and excessive food is being consumed. Indeed, it has been shown that sleep restricted subjects-in a setting of ad libitum access to palatable food-consume excessive amounts of calories from snacks [44].

\section{Conclusions}

We showed that prolonged sleep restriction in a situation that mimics a working week changes glucose metabolism and may lead to an increased risk of developing type 2 diabetes. Two nights of normal sleep, however, restored this effect. In addition, we showed that five nights of sleep restriction does not affect hunger feelings and results in elevated leptin levels. This suggests that sleep restriction per se as it would occur during a typical working week may not increase the risk of developing obesity. Therefore, the previously observed epidemiological associations between short sleep and obesity might be due to a common underlying factor rather than a direct causation between short sleep and obesity. In addition, the excessive consumption of calories from snacks rather than from meals during a period of restricted sleep may contribute to the development of weight gain and/or obesity [44].

\section{Acknowledgments}

This study was supported by the European Union Framework 6 (MCRTN-CT-2004-512362) and the Finnish Work
Environment Fund (FWEF; 104073 and 108203). The funders had no role in study design, data collection and analysis, decision to publish, or preparation of the manuscript. The authors thank Sirkku Hopeakangas for help with collection of blood samples, Marja-Leena Haavisto for the recruitment of participants, and Sirpa Hyttinen for help with cortisol analysis.

\section{References}

[1] E. Kronholm, T. Partonen, T. Laatikainen, et al., "Trends in self-reported sleep duration and insomnia-related symptoms in Finland from 1972 to 2005: a comparative review and re-analysis of Finnish population samples," Journal of Sleep Research, vol. 17, no. 1, pp. 54-62, 2008.

[2] S. M. W. Rajaratnam and J. Arendt, "Health in a 24-h society," Lancet, vol. 358, no. 9286, pp. 999-1005, 2001.

[3] S. R. Patel and F. B. Hu, "Short sleep duration and weight gain: a systematic review," Obesity, vol. 16, no. 3, pp. 643-653, 2008.

[4] B. Schultes, S. Schmid, A. Peters, J. Born, and H. L. Fehm, "Sleep loss and the development of diabetes: a review of current evidence," Experimental and Clinical Endocrinology and Diabetes, vol. 113, no. 10, pp. 563-567, 2005.

[5] K. Spiegel, R. Leproult, and E. Van Cauter, "Impact of sleep debt on metabolic and endocrine function," Lancet, vol. 354, no. 9188, pp. 1435-1439, 1999.

[6] K. Spiegel, E. Tasali, P. Penev, and E. Van Cauter, "Brief communication: sleep curtailment in healthy young men is associated with decreased leptin levels, elevated ghrelin levels, and increased hunger and appetite," Annals of Internal Medicine, vol. 141, no. 11, pp. 846-850, 2004.

[7] C. A. Everson and W. R. Crowley, "Reductions in circulating anabolic hormones induced by sustained sleep deprivation in rats," American Journal of Physiology, vol. 286, no. 6, pp. E1060-E1070, 2004.

[8] B. Bodosi, J. Gardi, I. Hajdu, E. Szentirmai, F. Obal Jr., and J. M. Krueger, "Rhythms of ghrelin, leptin, and sleep in rats: effects of the normal diurnal cycle, restricted feeding, and sleep deprivation," American Journal of Physiology, vol. 287, no. 5, pp. R1071-R1079, 2004.

[9] G. Jean-Louis, D. F. Kripke, S. Ancoli-Israel, M. R. Klauber, and R. S. Sepulveda, "Sleep duration, illumination, and activity patterns in a population sample: effects of gender and ethnicity," Biological Psychiatry, vol. 47, no. 10, pp. 921-927, 2000.

[10] W. M. A. van Leeuwen, M. Lehto, P. Karisola, et al., "Sleep restriction increases the risk of developing cardiovascular diseases by augmenting proinflammatory responses through IL-17 and CRP," PLoS ONE, vol. 4, no. 2, article e4589, 2009.

[11] J. E. Ferrie, M. J. Shipley, F. P. Cappuccio, et al., "A prospective study of change in sleep duration: associations with mortality in the Whitehall II cohort," Sleep, vol. 30, no. 12, pp. 16591666, 2007.

[12] D. J. Gottlieb, S. Redline, F. J. Nieto, et al., "Association of usual sleep duration with hypertension: the Sleep Heart Health Study," Sleep, vol. 29, no. 8, pp. 1009-1014, 2006.

[13] D. J. Gottlieb, N. M. Punjabi, A. B. Newman, et al., "Association of sleep time with diabetes mellitus and impaired glucose tolerance," Archives of Internal Medicine, vol. 165, no. 8, pp. 863-868, 2005.

[14] S. Stranges, F. P. Cappuccio, N.-B. Kandala, et al., "Crosssectional versus prospective associations of sleep duration with changes in relative weight and body fat distribution: the 
Whitehall II study," American Journal of Epidemiology, vol. 167, no. 3, pp. 321-329, 2008.

[15] B. Bjorvatn, I. M. Sagen, N. Oyane, et al., "The association between sleep duration, body mass index and metabolic measures in the Hordaland Health Study," Journal of Sleep Research, vol. 16, no. 1, pp. 66-76, 2007.

[16] H. K. Yaggi, A. B. Araujo, and J. B. McKinlay, "Sleep duration as a risk factor for the development of type 2 diabetes," Diabetes Care, vol. 29, no. 3, pp. 657-661, 2006.

[17] K. Spiegel, K. Knutson, R. Leproult, E. Tasali, and E. Van Cauter, "Sleep loss: a novel risk factor for insulin resistance and Type 2 diabetes," Journal of Applied Physiology, vol. 99, no. 5, pp. 2008-2019, 2005.

[18] R. Malouf and J. C. M. Brust, "Hypoglycemia: causes, neurological manifestations, and outcome," Annals of Neurology, vol. 17, no. 5, pp. 421-430, 1985.

[19] The DECODE Study Group, "Is the current definition for diabetes relevant to mortality risk from all causes and cardiovascular and noncardiovascular diseases?" Diabetes Care, vol. 26, no. 3, pp. 688-696, 2003.

[20] H.-P. Guler, J. Zapf, and E. R. Froesch, "Short-term metabolic effects of recombinant human insulin-like growth factor I healthy adults," New England Journal of Medicine, vol. 317, no. 3, pp. 137-140, 1987.

[21] R. Jacob, E. Barrett, G. Plewe, K. D. Fagin, and R. S. Sherwin, "Acute effects of insulin-like growth factor I on glucose and amino acid metabolism in the awake fasted rat. Comparison with insulin," Journal of Clinical Investigation, vol. 83, no. 5, pp. 1717-1723, 1989.

[22] E. J. Schoenle, P. D. Zenobi, T. Torresani, E. A. Werder, M. Zachmann, and E. R. Froesch, "Recombinant human insulinlike growth factor I (rhIGF I) reduced hyperglycaemia in patients with extreme insulin resistance," Diabetologia, vol. 34, no. 9, pp. 675-679, 1991.

[23] J. L. Leahy and K. M. Vandekerkhove, "Insulin-like growth factor-I at physiological concentrations is a potent inhibitor of insulin secretion," Endocrinology, vol. 126, no. 3, pp. 1593 1598, 1990.

[24] P. D. Zenobi, S. Graf, H. Ursprung, and E. R. Froesch, "Effects of insulin-like growth factor-I on glucose tolerance, insulin levels, and insulin secretion," Journal of Clinical Investigation, vol. 89, no. 6, pp. 1908-1913, 1992.

[25] T. L. S. Visscher and J. C. Seidell, "The public health impact of obesity," Annual Review of Public Health, vol. 22, pp. 355-375, 2001.

[26] K. L. Knutson and E. Van Cauter, "Associations between sleep loss and increased risk of obesity and diabetes," Annals of the New York Academy of Sciences, vol. 1129, pp. 287-304, 2008.

[27] J. Horne, "Short sleep is a questionable risk factor for obesity and related disorders: statistical versus clinical significance," Biological Psychology, vol. 77, no. 3, pp. 266-276, 2008.

[28] N. S. Marshall, N. Glozier, and R. R. Grunstein, "Is sleep duration related to obesity? A critical review of the epidemiological evidence," Sleep Medicine Reviews, vol. 12, no. 4, pp. 289-298, 2008.

[29] M. D. Klok, S. Jakobsdottir, and M. L. Drent, "The role of leptin and ghrelin in the regulation of food intake and body weight in humans: a review," Obesity Reviews, vol. 8, no. 1, pp. 21-34, 2007.

[30] Y. Zhang and P. J. Scarpace, "The role of leptin in leptin resistance and obesity," Physiology and Behavior, vol. 88, no. 3, pp. 249-256, 2006.

[31] K. Spiegel, R. Leproult, M. L'hermite-Baleriaux, G. Copinschi, P. D. Penev, and E. Van Cauter, "Leptin levels are dependent on sleep duration: relationships with sympathovagal balance, carbohydrate regulation, cortisol, and thyrotropin," Journal of Clinical Endocrinology and Metabolism, vol. 89, no. 11, pp. 5762-5771, 2004.

[32] S. Taheri, L. Lin, D. Austin, T. Young, and E. Mignot, "Short sleep duration is associated with reduced leptin, elevated ghrelin, and increased body mass index," PLoS Medicine, vol. 1, article e62, 2004.

[33] M. K. Sinha, J. Sturis, J. Ohannesian, et al., "Ultradian oscillations of leptin secretion in humans," Biochemical and Biophysical Research Communications, vol. 228, no. 3, pp. 733738, 1996.

[34] D. A. Schoeller, L. K. Cella, M. K. Sinha, and J. F. Caro, "Entrainment of the diurnal rhythm of plasma leptin to meal timing," Journal of Clinical Investigation, vol. 100, no. 7, pp. 1882-1887, 1997.

[35] R. V. Considine, M. K. Sinha, M. L. Heiman, et al., "Serum immunoreactive-leptin concentrations in normal-weight and obese humans," New England Journal of Medicine, vol. 334, no. 5, pp. 292-295, 1996.

[36] M. Korbonits, P. J. Trainer, J. A. Little, et al., "Leptin levels do not change acutely with food administration in normal or obese subjects, but are negatively correlated with pituitaryadrenal activity," Clinical Endocrinology, vol. 46, no. 6, pp. 751-757, 1997.

[37] P. W. Franks, I. S. Farooqi, J. Luan, et al., "Does physical activity energy expenditure explain the between-individual variation in plasma leptin concentrations after adjusting for differences in body composition?" Journal of Clinical Endocrinology and Metabolism, vol. 88, no. 7, pp. 3258-3263, 2003.

[38] M. Duclos, J.-B. Corcuff, A. Ruffie, P. Roger, and G. Manier, "Rapid leptin decrease in immediate post-exercise recovery," Clinical Endocrinology, vol. 50, no. 3, pp. 337-342, 1999.

[39] D. P. C. Van Aggel-Leijssen, M. A. Van Baak, R. Tenenbaum, L. A. Campfield, and W. H. M. Saris, "Regulation of average $24 \mathrm{~h}$ human plasma leptin level the influence of exercise and physiological changes in energy balance," International Journal of Obesity, vol. 23, no. 2, pp. 151-158, 1999.

[40] G. M. Lord, "Leptin as a proinflammatory cytokine," Contributions to Nephrology, vol. 151, pp. 151-164, 2006.

[41] G. Matarese, S. Moschos, and C. S. Mantzoros, "Leptin in immunology," Journal of Immunology, vol. 174, no. 6, pp. 3137-3142, 2005.

[42] C. B. Saper, T. C. Chou, and J. K. Elmquist, "The need to feed: homeostatic and hedonic control of eating," Neuron, vol. 36, no. 2, pp. 199-211, 2002.

[43] C. B. Saper, G. Cano, and T. E. Scammell, "Homeostatic, circadian, and emotional regulation of sleep," Journal of Comparative Neurology, vol. 493, no. 1, pp. 92-98, 2005.

[44] A. V. Nedeltcheva, J. M. Kilkus, J. Imperial, K. Kasza, D. A. Schoeller, and P. D. Penev, "Sleep curtailment is accompanied by increased intake of calories from snacks," American Journal of Clinical Nutrition, vol. 89, no. 1, pp. 126-133, 2009. 


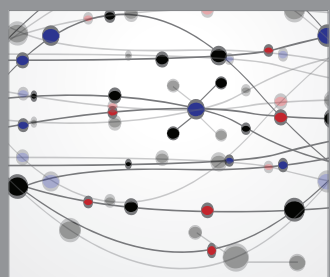

The Scientific World Journal
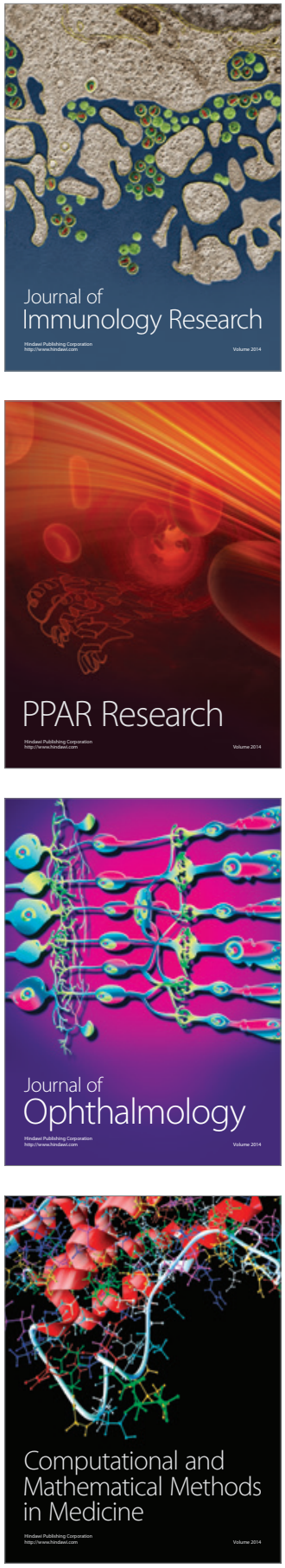

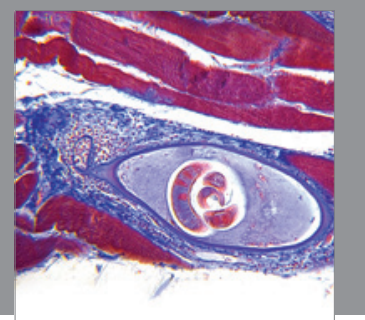

Gastroenterology

Research and Practice
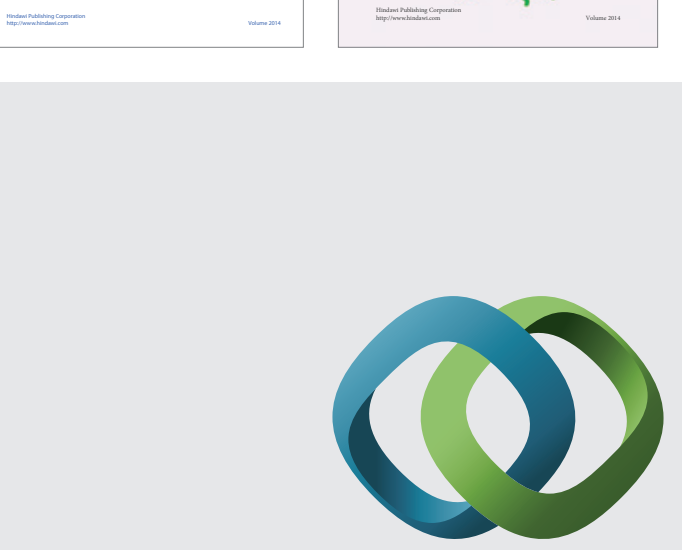

\section{Hindawi}

Submit your manuscripts at

http://www.hindawi.com
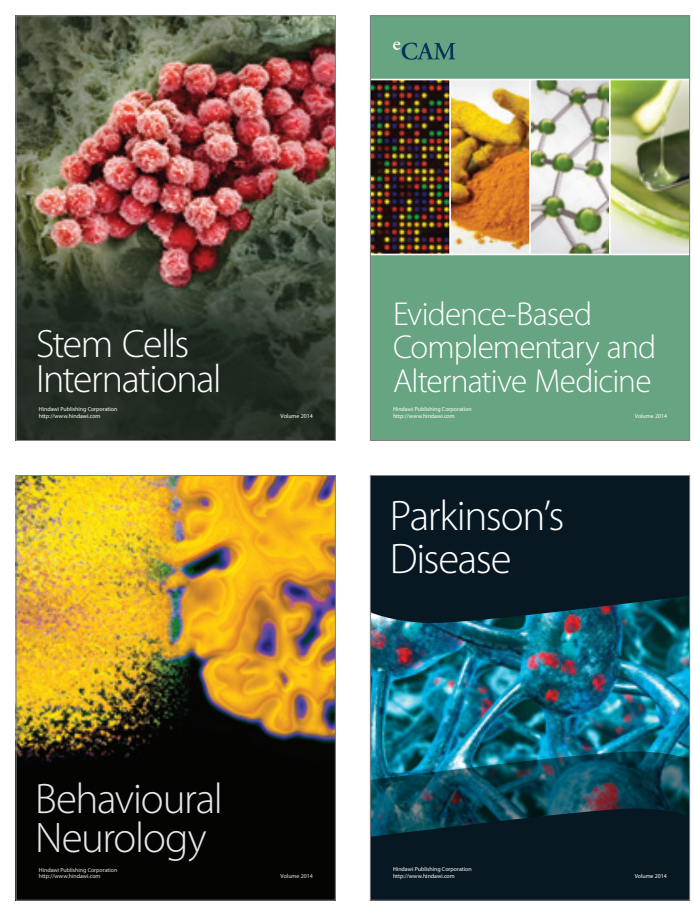

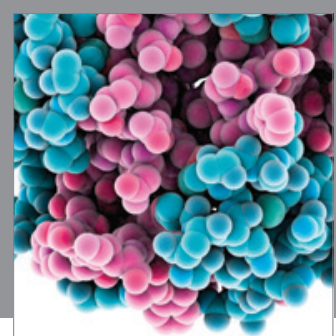

Journal of
Diabetes Research

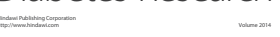

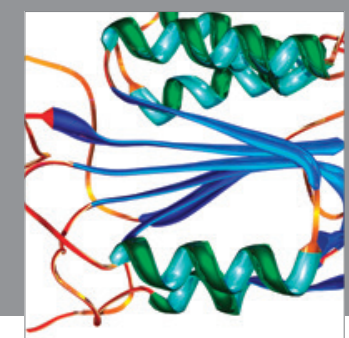

Disease Markers
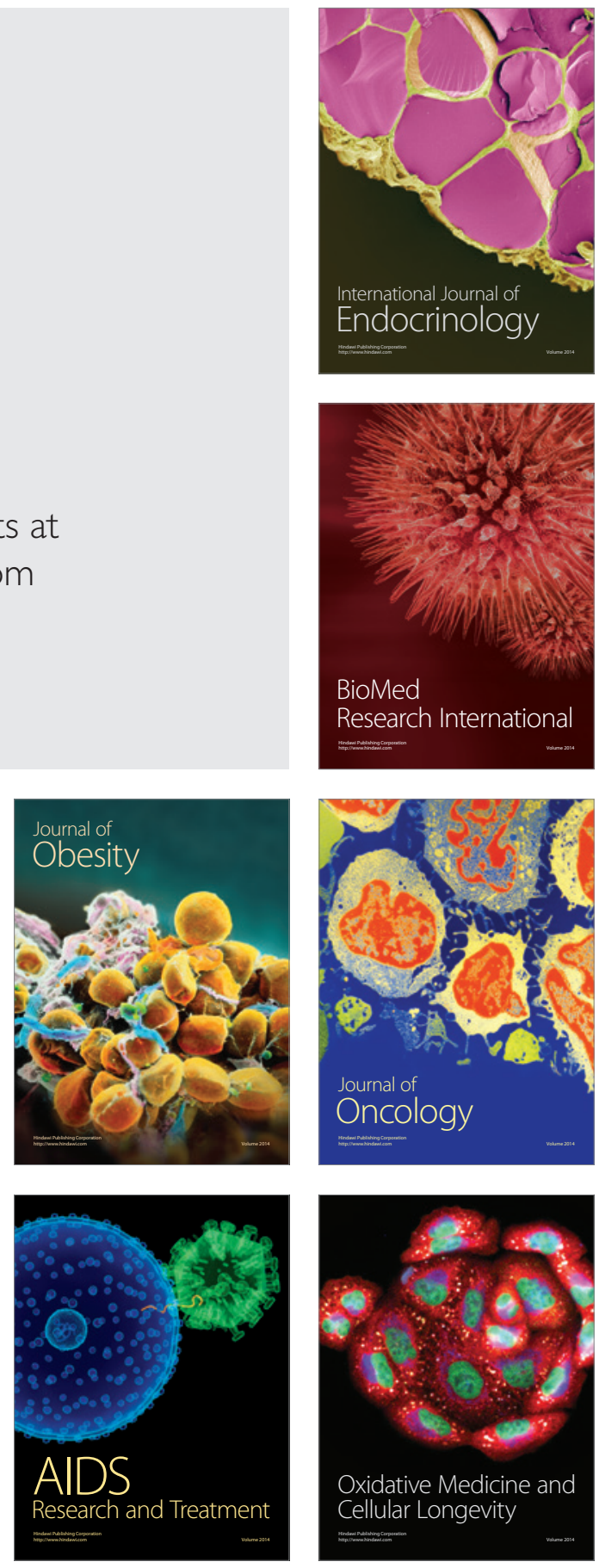